\title{
Coherent Charge Transport in Metallic Proximity Structures
}

\author{
A.A.Golubov ${ }^{a, b}$, F.K.Wilhelm ${ }^{c}$ and A.D.Zaikin ${ }^{c, d}$ \\ ${ }^{a}$ Institute of Thin Film and Ion Technology, Research Centre Jülich (KFA), D-52425 Jülich, Germany \\ ${ }^{b}$ Institute of Solid State Physics, 142432 Chernogolovka, Russia \\ ${ }^{c}$ Institut für Theoretische Festkörperphysik, Universität Karlsruhe, 76128 Karlsruhe, FRG \\ ${ }^{d}$ I.E.Tamm Department of Theoretical Physics, P.N.Lebedev Physics Institute, Leninskii prospect 53, 117924 Moscow, Russia
}

We develop a detailed microscopic analysis of electron transport in normal diffusive conductors in the presence of proximity induced superconducting correlation. We calculated the linear conductance of the system, the profile of the electric field and the densities of states. In the case of transparent metallic boundaries the temperature dependent conductance has a non-monotoneous "reentrant" structure. We argue that this behavior is due to nonequilibrium effects occuring in the normal metal in the presence of both superconducting correlations and the electric field there. Low transparent tunnel barriers suppress the nonequilibrium effects and destroy the reentrant behavior of the conductance. If the wire contains a loop, the conductance shows Aharonov-Bohm oscillations with the period $\Phi_{0}=h / 2 e$ as a function of the magnetic flux $\Phi$ inside the loop. The amplitude of these oscillations also demonstrates the reentrant behavior vanishing at $T=0$ and decaying as $1 / T$ at relatively large temperatures. The latter behavior is due to low energy correlated electrons which penetrate deep into the normal metal and "feel" the effect of the magnetic flux $\Phi$. We point out that the density of states and thus the "strengh" of the proximity effect can be tuned by the value of the flux inside the loop. Our results are fully consistent with recent experimental findings.

\section{INTRODUCTION}

Recent progress in nanolithographic technology revived the interest to both experimental and theoretical investigation of electron transport in various mesoscopic proximity systems consisting of superconducting and normal metallic layers. In such systems the Cooper pair wave function of a superconductor penetrates into a normal metal at a distance which increases with decreasing temperaturet. At sufficiently low temperatures this distance becomes large and the whole normal metal may acquire superconducting properties. Although this phenomenon has been already understood more than thirty years ago and intensively investigated during past decades, recently novel physical features of metallic proximity systems have been discovered 18 and studied theoretically (see 21 and further references therein).

In this paper we study the influence of the proximity effect on transport properties of a diffusive conductor in the limit of relatively low temperatures and voltages. We will assume that this conductor is brought in a direct contact to a superconducting reservoir which serves as an effective injector of Cooper pairs into a normal metal. We will show that if the system contains no tunnel barriers there are two different physical regimes which determine the system conductance in different temperature intervals. It is well known that proximity induced superconducting correlation between electrons in a diffusive normal metal survives at a distance of order $\xi_{N} \sim \sqrt{\mathcal{D} / T}$, where $\mathcal{D}=v_{F} l_{i m p} / 3$ is the diffusion coefficient. As $T$ is lowered the proximity induced superconductivity expands into the normal metal and, consequently, the "normally conducting" part of the system effectively shrinks in size. This effect results in increasing of the conductance of a normal metal. At sufficiently low temperature the length $\xi_{N}$ becomes of order of the size of the normal layer and the system behavior becomes sensitive to a physical choice of the boundary condition at the edge of the normal wire opposite to that attached to a superconductor.

One possible choice of this boundary condition corresponds to the assumption that a nontransparent barrier is present at the edge of this wire. Then electrons cannot diffuse out of the wire, the proximity induced superconducting correlation survives everywhere in the system and a real gap in the quasiparticle spectrum develops in the N-metal22. The value of this gap is of order of $\varepsilon_{g} \sim \min \left(\Delta, \mathcal{D} / L^{2}\right), \Delta$ is the bulk superconducting gap and $L$ is the length of the normal wire.

Another possible situation corresponds to the presence of a big normal reservoir N' directly attached to the Nwire by means of a highly transparent contact. In this case even at very low $T$ the proximity induced Cooper pair amplitude is essentially nonhomogeneous in the $\mathrm{N}$ metal. Indeed, close to a superconductor this amplitude is large, whereas in the vicinity of a normal reservoir it is essentially suppressed. Thus, strictly speaking, the whole $\mathrm{N}$-wire cannot be characterized by the real gap in its quasiparticle spectrum. In the absense of a potential barrier between $\mathrm{N}$ and $\mathrm{N}^{\prime}$ this gap is obviously equal to zero at the $\mathrm{NN}^{\prime}$ interface and - as will be demonstrated - everywhere in the normal metal. Nevertheless, it turns out that the density of states in the N-metal shows a soft pseudogap which is again of the order of $\varepsilon_{g}$. In other words, the spacially averaged normalized density of states $N_{N}(\varepsilon)$ in the N-wire at small $\varepsilon \lesssim \varepsilon_{d}$ is smaller than its normal state value $N_{N}<N(0)$ but always remains nonzero. It increases with increasing $\varepsilon$ and reaches the value $N_{N}=N(0)$ at $\varepsilon \gtrsim \varepsilon_{d}$. This is the key point for understanding the low temperature behavior of the conductance of our system. As the temperature increases from zero, higher and higher values of 
$\varepsilon$ contribute to the current and the system conductance - due to the increase of $N_{N}$ with $\varepsilon$ - increases with $T$. This regime takes place until the temperature reaches the value $T \sim \varepsilon_{d}$ where the crossover to a high temperature behavior takes place. Note that similar behavior of the normal metal conductance in the presence of proximityinduced superconductivity has been recently found by Nazarov and Stoot23.

An interesting feature of the system without tunnel barriers is that at $T=0$ its conductance exactly coincides with that of a normal metal with no proximity effects. This result has been first obtained by Artemenko, Volkov and Zaitsev24 for the case of a normalsuperconducting constriction. Although it is already around for many years the physical meaning of this result - if any - still needs to be understood. At the first sight the linear conductance of the system at $T=0$ should be smaller than in the normal state because of the presence of the (pseudo)gap in the normal DOS $N_{N}$ at low energies. Why is this not the case?

In order to answer this question we should recall the well known fact that in the presence of nonequilibrium effects the current flowing in a superconductor depends not only on the normal DOS but is characterized by a set of generalized DOS25. Our problem is just a particular example of a nonequilibrium superconductor: on one hand superconducting correlation penetrates into the normal metal and the Cooper pair amplitude is nonzero there, on the other hand in the absence of low transparent tunnel barriers the electric field also penetrates into the N-metal and drives the quasiparticle distribution function out of equilibrium. We will argue that in this situation one of the generalized DOS (below we define it as $N_{S}(\varepsilon)$ ) - that is nonzero in the N-layer due to the presence of proximity induced superconducting correlation at low energies plays an important role and also contributes to the system conductance. In other words, in the presence of the electric field inside the system both uncorrelated and correlated electrons contribute to a dissipative current. This is the reason why in the presence of proximity induced superconductivity the system conductance is never smaller than its normal state value although the normal DOS $N_{N}(\varepsilon)<N(0)$ at low energies 26 .

We would like to emphasize that the situation is entirely different in the presence of low transparent tunnel barriers. Provided their resistances are much larger than that of the N-metal the whole voltage drop takes place at these barriers and the electric field does not penetrate into the N-layer. In this case only uncorrelated electrons contribute to the dissipative current and therefore only the normal DOS $N_{N}$ matters. As a result the temperature dependence of the system conductance changes. We will demonstrate that with lowering the barrier transparency the crossover takes place to the effective conductance decreasing monotonously with $\mathrm{T}$, characteristic for two serial NIS' tunnel junctions ( $S$ ' is now the diffusive normal conductor with the proximity-induced gap).

Note that both types of the behavior, namely reen- trant and monotonously decreasing with $T$ conductance have been observed in the experiments 3 , 8 . Furthermore, we would like to point out that both densities of states $N_{N}(\varepsilon)$ and $N_{S}(\varepsilon)$ can be investigated in one experiment. We will come back to this point further below.

When the system contains a mesoscopic loop of a normal metal, the conductance as a function of the magnetic flux through the loop shows oscillations with the period $\Phi_{0}=h / 2 e$ (superconducting flux quantum). Although the Cooper pair amplitude (and thus the supercurrent) in the ring is exponentially small at $T \gg \epsilon_{d}$, the amplitude of these oscillations decays only as $\propto 1 / T$. This again illustrates an important difference in the behavior of kinetic (conductance) and thermodynamic (supercurrent) quantities. Below we will argue that in the systems considered here even at large $T \gg \epsilon_{d}$ the behavior of the first quantity is dominated by correlated low energy electrons with $\epsilon \lesssim \epsilon_{d}$ penetrating far into the normal metal whereas the contribution of electrons with $\epsilon \sim T$ to the second one is only important. Again the presence of the electric field inside the $\mathrm{N}$-metal is crucially important for this effect. At low $T$ the oscillation amplitude again shows the reentrant behavior and vanishes in the limit $T \rightarrow 0$ as $T^{2}$. A clear experimental evidence for a $1 / T$ decay of the conductance oscillations has been recently reported in Ref. 6.

Finally we point out that making use of the the geometry with a metallic loop one can easily tune the densities of states of the system by applying a magnetic flux $\Phi$ inside this loop. We will show that e.g. for $\Phi=\Phi_{0} / 2$ the proximity effect in the normal region "after" the loop is completely suppressed and the normal $\operatorname{DOS} N_{N}(\epsilon)=N(0)$ there. This effect can be investigated experimentally and used for further studies of proximity induced superconductivity in normal metullic structures.

The structure of our paper is as follows. In Section II we briefly describe the general kinetic approach based on quasiclassical Green functions in the Keldysh technique and define the physical quantities of interest. Then a detailed analysis of this quantities (conductance - Section III, DOS - Section IV, elecric field - Section V) will be presented. Sections VI and VII are devoted to the extension of our analysis to the proximity systems containing mesoscopic normal metal loops with a magnetic flux. The main results of the present paper are summarized in Section VIII. Further details related to different geometric realizations of the proximity systems with loops are presented in Appendix.

\section{KINETIC ANALYSIS}

\section{A. General formalism}

Let us consider a quasi-one-dimensional normal conductor of a length $2 L$ with a superconducting strip of a thickness $2 d_{s}$ attached to a normal metal on the top of 
it and two normal reservoirs attached to its edges (see fig.1). The length $L$ is assumed to be much larger than the elastic mean free path $l_{i m p}$ but much shorter than the inelastic one. This geometrical realization has afirect re-

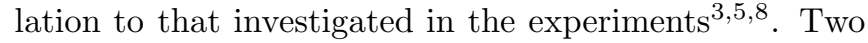
big normal reservoirs N' are assumed to be in thermodynamic equilibrium at the potentials $V$ and 0 respectively. In contrast to the case of a ballistic constriction 2728 the potential drop within the system is distributed between the interfaces and the conductor itself. The general approach to calculate conductance of these structures was developed in 910,12 . In what follows we shall apply this method to analyse the temperature dependence of the NS proximity structure of fig. 11.

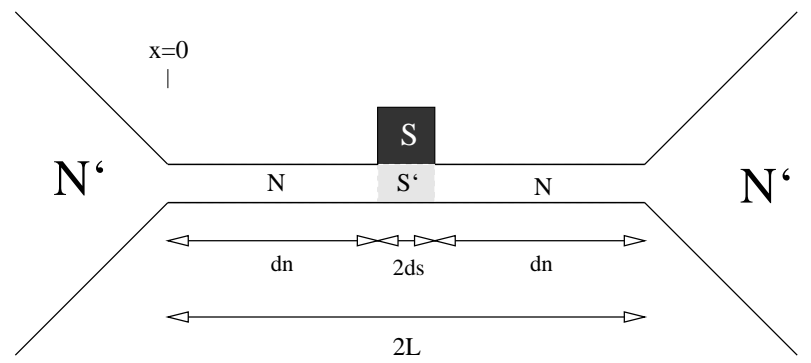

FIG. 1. The experimental system under consideration

Such an experimental realization allows to prepare a structure without effective tunnel barriers in the direction of the current flow. Even with "perfect" samples in a usual sandwich geometry, a natural barrier shows up due to the inevitable mismatch of Fermi velocities between different materials. This could well be one of the reasons why in the previous experiments with sandwichlike structures, the reentrant behavior of the conductance was not detected 29 .

The electron transport through the metallic system can be described by the equations for a matrix of quasiclassical Green functions $\stackrel{\vee}{G}$ in the contact 30,31 .

$$
\stackrel{\vee}{G}=\left(\begin{array}{cc}
\hat{G}^{R} & \hat{G}^{K} \\
0 & \hat{G}^{A}
\end{array}\right)
$$

where $\hat{G}^{A}, \hat{G}^{R}$ and $\hat{G^{K}}$ are respectively the impurityaveraged advanced, retarded and Keldysh Green functions. These functions are in turn matrices in the Nambu space:

$\hat{G}^{R}=\hat{\sigma}_{z} g^{R}+i \hat{\sigma}_{y} f^{R}, \hat{G}^{A}=-\left(\hat{G}^{R}\right)^{*}$ and $\hat{G}^{K}=\hat{G}^{R} \hat{f}-\hat{f} \hat{G}^{A}$

Here the distribution function $\hat{f}=f_{l}+\hat{\sigma}_{z} f_{t}$, where $f_{l}=\tanh (\varepsilon / 2 T)$ and $f_{t}$ describes deviation from equilibrium. Taking advantage of the normalization condition for the normal and the anomalous Green functions $\left(g^{R}\right)^{2}-\left(f^{R}\right)^{2}=1$ it is convenient to parametrize $g^{R}=\cosh \theta, f^{R}=\sinh \theta$, where $\theta \equiv \theta_{1}+i \theta_{2}$ is a complex function. Deep in the bulk superconductor it is equal to $\theta_{s}=1 / 2 \ln [(\Delta+\varepsilon) /(\Delta-\varepsilon)]-i \pi / 2$ for $\varepsilon<\Delta$ and $\theta_{s}=(1 / 2) \ln [(\varepsilon+\Delta) /(\varepsilon-\Delta)] / 2$ for $\varepsilon>\Delta$ (here and below we omit the indices $\mathrm{R}(\mathrm{A}))$.

The current $I$ and the electrostatic potential $\phi$ are expressed through $\stackrel{\vee}{G}$ as

$$
\begin{gathered}
I=\frac{\nu \mathcal{D} S}{2} \int_{-\infty}^{\infty} d \varepsilon S p\left[\hat{\sigma}_{z} \stackrel{\vee}{G} \partial_{x} \stackrel{\vee}{G}\right]^{K}, \\
\phi(x)=\int_{0}^{\infty} d \varepsilon \operatorname{Tr} \hat{g}^{K}(x, \varepsilon)=\int_{0}^{\infty} d \varepsilon f_{t}(x, \varepsilon) \nu_{\varepsilon}(x),
\end{gathered}
$$

where $\nu$ is the the density of states, $\nu_{\varepsilon}(x)=\Re\left(g_{\varepsilon}^{R}(x)\right)$ and $S$ is the crossection area of $N$ conductor.

Being expressed in terms of the function $\theta(\varepsilon, x)$ the equation 30,31 for the Green functions and the distribution function for the N-metal take a particularly simple form

$$
\begin{gathered}
\mathcal{D} \partial_{x}^{2} \theta+2 i \varepsilon \sinh \theta=0 \\
\partial_{x}\left[\mathcal{D}\left(\cosh ^{2} \theta_{1}\right) \partial_{x} f_{t}\right]=0,
\end{gathered}
$$

$x$ is the coordinate along the $\mathrm{N}$-conductor. Here we neglected the processes of inelastic relaxation and put the pair potential in the normal metal equal to zero $\Delta_{N}=0$ assuming the absence of electron-electron interaction there.

Before we come to a detailed solution of the problem let us point out that the conclusion about the anomalous behavior of the system conductance can be reached already from the form of eq. (5). Indeed it is quite clear from (5) that the effective diffusion coefficient $\mathcal{D}$ eff $=\mathcal{D} \cosh ^{2} \theta_{1}$ increases in the N-regions with proximity-induced superconductivity and, therefore, the electric field is partially expelled from these regions. This energy dependent field modulation is controlled by the solution for $\theta(\varepsilon, x)$ and is directly related to the physical origin of the anomalous temperature dependence of the system conductance discussed below.

The equations (4) and (5) should be supplemented by the boundary conditions at the interfaces of the normal metal N. Assuming that the anomalous Green fupction of big normal reservoirs $N$ ' is equal to zero from 32,10 we obtain

$$
\begin{gathered}
\xi_{N}^{*} \gamma_{B} \partial_{x} \theta= \pm \sinh \theta \\
\xi_{N}^{*} \gamma_{B} \cosh \theta_{1} \partial_{x} f_{t}= \pm \cosh \theta_{2}\left(f_{t}-f_{t}(x=0,2 L)\right)
\end{gathered}
$$

where $\gamma_{B}=R_{b} / \rho_{N} \xi_{N}^{*}$ is the interface resistance parameter, $R_{b}$ is the specific resistance of the interface between the N-conductor and the N'-reservoirs, $\rho_{N}$ is the resistivity of the N-metal, and $\xi_{N}^{*}=\sqrt{D_{N} / 2 \pi T_{c}}$ is the temperature independent characteristic length scale in $\mathrm{N}$ (note that the coherence length in $\mathrm{N} \xi_{N}(T)=\sqrt{D_{N} / 2 \pi T}$ is T-dependent). 
In general we should also fix the boundary condition at the interface between the N-metal and the superconductor. For the case of a perfect transparency of this interface and for typical thickness of the normal layer $w_{N} \sim \sqrt{S}$, Cooper pairs easily penetrate into it due to the proximity effect and the Green functions of the Nmetal at relatively low energies for $d \leq x \leq d+2 d_{s}$ are equal to those of a bulk superconductor $\theta=\theta_{s}$ (the influence of finite transparency of the NS-contact will be discussed below). In this sence the region of a normal metal situated directly under the superconductor can be also treated as a piece of a superconductor $S^{\prime}$ and the solution of (4), (5) needs to be found only for $0<x<d$ (without loss of generality we will stick to a symmetric configuration).

Cooper pairs penetrate into the normal conductor also in the case of a not perfectly transparent NS interface. As it is demonstrated below, the energy gap is induced in S' region in this case. As a result, for a sufficiently long $\mathrm{N}$-wire, which is only considered here, the presence of the barrier at the NS interface will not influence the results derived for the system conductance.

\section{B. Physical quantities of interest}

Prpceeding along the same lines as it has been done in ref 12 we arrive at the final expression for the current

$I=\frac{1}{2 R} \int_{0}^{\infty} d \varepsilon\left[\tanh \left(\frac{\varepsilon+e V}{2 T}\right)-\tanh \left(\frac{\varepsilon-e V}{2 T}\right)\right] D(\varepsilon)$,

where $D(\varepsilon)$ defines the effective transparency of the system 12

$D(\varepsilon)=\frac{1+r}{\frac{r}{\cosh \theta_{1}(x=0, \varepsilon) \cos \theta_{2}(x=0, \varepsilon)}+\frac{1}{L} \int_{0}^{L} d x \operatorname{sech}^{2} \theta_{1}(x, \varepsilon)}$,

$R=R_{b}+R_{N}$ and $r=R_{b} / R_{N} \equiv \gamma_{B} \xi_{N}^{*} / L, R_{N}$ is the resistance of the $\mathrm{N}$-metal.

Let us consider the case of a sufficiently long normal conductor $d^{2} \gg \mathcal{D} / \Delta$. Then at low temperatures $T \ll \Delta$ the interesting energy interval is restricted to $\varepsilon \ll \Delta$. For such values of $\varepsilon$ the contribution of the $S^{\prime}$-part of the normal conductor shows no structure and can be easily taken into account with the aid of obvious relations

$$
\int_{0}^{L} d x \operatorname{sech}^{2} \theta_{1}(x, \varepsilon)=\int_{0}^{d} d x \operatorname{sech}^{2} \theta_{1}(x, \varepsilon)+d_{s} \operatorname{sech}^{2} \theta_{s, 1}
$$

and $\operatorname{sech}^{2} \theta_{s, 1}=\left(1-\frac{\varepsilon^{2}}{\Delta^{2}}\right)$ (no barrier at the NS interface) or $\operatorname{sech}^{2} \theta_{s, 1}=\left(1-\frac{\varepsilon^{2}}{\Delta_{g N}^{2}}\right)$ (the barrier is present at the NS interface). Due to this reason we will discuss only the properties of the $N$-part $(0<x<d)$. For the sake of completeness we will also demonstrate the effect of finite $d_{s}$ in the end of our calculation.

For the differential conductance of the $N$-part $0 \leq x \leq$ $d$ normalized to its normal ("non-proximity") value in the zero bias limit eq. (7) yields

$$
\bar{G}_{N}=\left(\frac{R d I}{d V}\right)_{V=0}=\frac{1}{2 T} \int_{0}^{\infty} d \varepsilon D(\varepsilon) \operatorname{sech}^{2}(\varepsilon / 2 T) .
$$

Analogously the normalized zero-bias electrostatic potential distribution reads

$$
\begin{aligned}
\phi_{0}(x)=\lim _{V \rightarrow 0} \frac{\phi(x)}{V}= & \frac{1}{2 T d} \int_{0}^{\infty} d \varepsilon D(\varepsilon) \nu_{\varepsilon}(x) \operatorname{sech}^{2}(\varepsilon / 2 T) \times \\
& \times \int_{x}^{d} d x^{\prime} \operatorname{sech}^{2}\left(\theta_{1}\left(x^{\prime}\right)\right)
\end{aligned}
$$

The normal density of states is given by the normal Green's function via the standard relation $\nu_{\epsilon}(x)=$ $N(0) \Re\left(g_{\epsilon}(x)\right)$ which enters into the conductance in the form $\cosh ^{2} \theta_{1}=(\Re g)^{2}+(\Im f)^{2}$ together with a 'correlation DOS' $\eta_{\epsilon}(x)=N(0) \Im\left(f_{\epsilon}(x)\right)$. The importance of the latter quantity for understanding the effects discussed here has been already pointed out in the Introduction. We will discuss the features of these local densities as well as the averaged ones:

$$
\begin{aligned}
N_{N}(\epsilon) & =\int d \bar{x} \nu_{\epsilon}(\bar{x}) \\
N_{S}(\epsilon) & =\int d \bar{x} \eta_{\epsilon}(\bar{x})
\end{aligned}
$$

As it has been already mentioned the "correlation DOS" $\eta$ belongs to the set of generalized densities of states familiar from the standard theory of nonequilibrium superconductivity 25,34. It reflects the presence of superconducting correlations at low energies. E.g. in a BCS superconductor this function reads $\eta=\frac{\Delta \Theta(\Delta-\epsilon)}{\sqrt{\Delta^{2}-\epsilon^{2}}}$. In our case this function is not only energy- but also space-dependent due to the fact that the proximity induced superconducting correlation decays inside the normal metal. But the physical meaning of it remains the same as in standard nonequilibrium superconductivity theory 25: $\eta$ plays a role whenever the quasiparticle distribution function of a superconductor is driven out of equilibrium. It happeps e.g. in the well-known problems of charge relaxation 34 and imbalance 35 . It happens also here due to a simultaneous presence of the electric field and the proximity induced superconducting correlation in the normal metal.

\section{Influence of finite barrier transparency at the top NS-interface}

Let us consider the effect of a tunnel barrier at the NS interface in more details. 
Under the assumption that the $\mathrm{N}$-wire thickness is small $w_{N} \ll \xi_{N}^{*}$ the equation for $\theta$ in the region $0 \leq$ $x \leq 2 d_{s}$ underneath the superconducting terminal can be derived by the method of Ref.36.

$$
\mathcal{D} \partial_{x}^{2} \theta+2 i \widetilde{\varepsilon} \sinh \theta+\widetilde{\Delta} \cos \theta=0
$$

where the effective order order parameter $\widetilde{\Delta}=$ $\sin \theta_{s} / \gamma_{B}^{N S}$, the effective energy $\widetilde{\varepsilon}=\varepsilon+\cos \theta_{s} / \gamma_{B}^{N S}$, $\gamma_{B}^{N S}=\left(R_{b} / \rho_{N}\right)\left(w_{N} / \xi_{N}^{* 2}\right)$ is the interface transparency parameter. Here $\theta_{s}$ is the solution in $\mathrm{S}$ which is set equal to the bulk value $\tan ^{-1}(i \Delta / \varepsilon)$, a good approximation for thin $\mathrm{N}$ film $w_{N} \ll \xi_{N}^{*}$. With these substitutions the equation (12) in the $\mathrm{N}$ film has the form similar to that in a superconductor. This equation is valid for $\gamma_{B}^{N S}>\left(w_{N} / \xi_{N}^{*}\right)^{2}$ i.e. for sufficiently small transparency of the NS interface: $\langle D\rangle<l_{N} / w_{N}$.

As follows from Eq.(12), superconducting properties of the N-layer are described in terms of the energydependent coherence length

$$
\xi_{N}(\epsilon)=\left\{\hbar D_{N} / 2\left[\gamma_{B}^{-2}-\epsilon^{2}-2 i \gamma_{B}^{-1} \epsilon \cos \theta_{S}\right]^{1 / 2}\right\}^{1 / 2}
$$

which determines an exponential decay of $N_{N}(x, \epsilon)$ with $x$.

The expression for $\xi_{N}(\epsilon)$ has a pole at the gap energy $\epsilon=\Delta_{g N}$, which signals the decay of quasiparticles entering $\mathrm{N}$ at $\epsilon<\Delta_{g N}$. At high energy $\epsilon \gg \pi T_{c} / \gamma_{B}^{N S}$ the well known result $\xi_{N}(\epsilon)=\left(\hbar D_{N} / 2 \epsilon\right)^{1 / 2}$ is reproduced, whereas at low energies $\epsilon \ll \pi T_{c} / \gamma_{B}^{N S}$ one obtains $\xi_{N}=\left(\hbar D_{N} \gamma_{B}^{N S} / 2 \pi T_{c}\right)^{1 / 2}$. Thus the effective length scale in $\mathrm{N}$ increases with the decrease of the SN interface transparency.

It is straightforward to calculate the gap energy $\Delta_{g N}$ assuming the "rigid" boundary conditions $\theta_{s}=$ $\tan ^{-1}(i \Delta / \varepsilon)$ either from the pole of $\xi_{N}(\epsilon)$ or, making use of the solution $\theta=\tanh ^{-1}\left[\sinh \theta_{s} /\left(\cosh \theta_{s}-i \varepsilon \gamma_{B}^{N S}\right)\right]$ and calculating the quasiparticle density of states $N_{N}(\varepsilon)=R e \cosh \theta$. Subsituting the expression for $\theta_{s}$ into this solution one arrives the equation for the energy gap

$$
t^{3}+2 C t^{2}+\left(C^{2}-1\right) t-2 C=0
$$

where $t=\sqrt{1-\left(\Delta_{g N} / \Delta\right)^{2}}$ and $C=\pi T_{c} / \gamma_{B}^{N S} \Delta$. The general solution is rather cumbersome, therefore here we present only its asymptotic forms.

The gap is given by $\Delta_{g N} / \Delta=1-2\left(\gamma_{B}^{N S} \Delta / \pi T_{c}\right)^{2}$ for large transparency of the NS interface, $\gamma_{B}^{N S} \Delta / \pi T_{c} \ll 1$, and by $\Delta_{g N}=\Delta /\left(1+\gamma_{B}^{N S} \Delta / \pi T_{c}\right)$ for small transparency, $\gamma_{B}^{N S} \Delta / \pi T_{c} \gg 1$. In the latter case (the McMillan limit), the expression for the gap may be written as $\Delta_{g N}=\pi T_{c} / \gamma_{B}^{N S} \equiv \hbar v_{F N}\langle D\rangle / 4 w_{N}$. The gap originates from the finite average lifetime $\tau_{N}=2 w_{N} /\langle D\rangle v_{F N}$ for quasiparticles in the $\mathrm{N}$ layer with respect to Andreev scattering from $\mathrm{S}$, since a contribution of gapless quasiparticle trajectories parallel to the NS interface is eliminated in the diffusive regime. The dependence of $\Delta_{g N}$ on $\gamma_{B}^{N S}$ in the whole range of transparencies is presented in Fig.2. Note that under a substitution $w_{N} \overline{\bar{B}} \pi / k_{F}$ the above expressions reproduce the result of Ref. 33 for a gap induced in a $2 \mathrm{D}$ electron gas in contact to a superconductor. We also note that in the case of two superconducting terminals attached to $\mathrm{N}$ the gap aquires a phase factor $\Delta_{g N} \rightarrow \cos (\varphi / 2) \Delta_{g N}, \varphi$ being the phase difference between the terminals.

At the subgap energies $\varepsilon \leq \Delta_{g N}$ the solution in $\mathrm{N}$ is $\theta \simeq 1 / 2 \ln \left[\left(\Delta_{g N}+\varepsilon\right) /\left(\Delta_{g N}-\varepsilon\right)\right]-i \pi / 2$ whereas above the gap, at $\varepsilon>\Delta_{g N}, \theta \simeq 1 / 2 \ln \left[\left(\varepsilon+\Delta_{g N}\right) /\left(\varepsilon-\Delta_{g N}\right)\right]$, in complete analogy with the solutions in a bulk superconductor but with reduced gap. Therefore the results for conductance of $\mathrm{N}$ depend on the relation between the gap $\Delta_{g}$ and the Thouless energy $\varepsilon_{d}=D / d^{2}$. Since for the case of a sufficiently long $\mathrm{N}$-wire, which is only considered in this paper, the condition $\Delta_{g N} \gg \varepsilon_{d}$ is satisfied, the presence of the barrier at the NS interface does not influence our results for the system conductance.

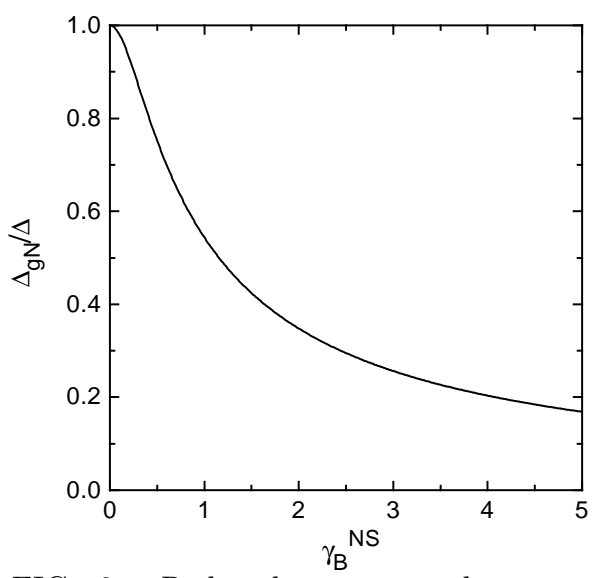

FIG. 2. Reduced gap on the normal side of the $N S$-boundary in the presence of barriers

\section{CONDUCTANCE}

\section{A. Perfectly transparent boundaries}

The analysis of the problem can be significantly simplified in the case of perfectly transparent interfaces $\left(\gamma_{B}=0\right)$. In this case the boundary conditions are

$$
\begin{aligned}
& \theta(0)=0 \\
& \theta(d)=\theta_{S}
\end{aligned}
$$

for the contact to the normal and the superconducting reservoir respectively. The effective transparency of the $\mathrm{N}$-part then reads

$$
D(\varepsilon)=\left(\frac{1}{d} \int_{0}^{d} d x \operatorname{sech}^{2}\left(\theta_{1}(x)\right)\right)^{-1} .
$$


As it was already pointed out for relatively long normal conductors and at low $T$ only the energies $\varepsilon \ll \Delta$ give an important contribution to the conductance. In this case the typical energy scale is defined by the Thouless energy $\epsilon_{d}=\mathcal{D} / d^{2} \ll \Delta, \Delta_{g N}$. For these energies we can set $\theta_{S}=-i \pi / 2$. Let us first put $T=0$. Then the thermal distribution factor $\operatorname{sech}^{2}(\varepsilon / 2 T) /(2 T)$ reduces to a delta function and we have

$$
\bar{G}_{N}(T=0)=D(0),
$$

i.e. we only need the solution of (4) with boundary conditions (15) at $\varepsilon=0$, which is $\theta=-i \frac{\pi}{2} \bar{x}$. This does not depend on $\mathcal{D}$, so the correlations are destroyed by the influence of the boundary conditions but not by thermal excitation or by impurity scattering. From here, we can calculate the conductance

$$
\bar{G}_{N}(T=0)=1,
$$

i.e. at $T=0$ the system conductance exactly coincides with its normal state value (cf.24.23. This result, however, by no means implies the destruction of the proximity induced superconductivity in the N-layer. Later on, we will demonstrate, that the DOS and the electrical field are completely different from their values in the normal state and in fact only due the additional contribution of correlated electrons the system conductance does not fall below its normal state value.

In the case $T \ll \varepsilon_{d}$ we can calculate $\theta$ perturbatively. From $\mathcal{D} \partial_{x}^{2} \theta=-2 i \varepsilon \sinh \theta_{0}(x)$ and (15) we get

$$
\theta=-\frac{8}{\pi^{2}} \frac{\varepsilon}{\varepsilon_{d}}[\bar{x}-\sin (\bar{x} \pi / 2)]-i \frac{\pi}{2} \bar{x} .
$$

Keeping only leading order terms in $\frac{\epsilon}{\epsilon_{d}}$, we get

$$
\bar{\sigma}_{N}=1+A \frac{T^{2}}{\epsilon_{d}^{2}},
$$

where $A=\frac{64}{3 \pi^{4}}\left(\frac{5}{6}-\frac{8}{\pi^{2}}\right) \approx 0.049$ is a universal constant. This means, that for low temperatures $\bar{G}_{N}(T)$ grows quadratically on the scale of $\varepsilon_{d}$ and approaches the crossover towards the high temperature regime discussed below.

In the limit $T \gg \epsilon_{d}$ (where we still have $T \ll \Delta$ ), the contribution of the low energy components to the thermally weighted integral for $\bar{G}_{N}(T)$ is $\propto 1 / T$ as we will see below and can therefore be neglected. We only have to take into account the solptions of (4) for energies $\epsilon \gg \epsilon_{d}$. It is well known (see e.g.12), that for this energy range the solution of (4) together with (15) reads

$$
\tanh (\theta(\bar{x}) / 4)=\tanh \left(\frac{i \pi}{8}\right) e^{k(\bar{x}-1)}
$$

where $k=d \sqrt{-2 i \varepsilon / \mathcal{D}}$. By using obvious substitutions and multiple-argument relations for hyperbolic functions, we arrive at the following identity: $\int_{\bar{x}}^{1} d \bar{x} \operatorname{sech}^{2}\left(\theta_{1}(\bar{x})\right)=(1-\bar{x})-4 \sqrt{\frac{\varepsilon_{d}}{\varepsilon}} \int_{0}^{\Re(k)(1-\bar{x})} \frac{q(y) d y}{(1+q(y))^{2}}$

where $q(y)=4(3+2 \sqrt{2}) e^{-2 y} \sin ^{2} y /\left(e^{-2 y}+3+2 \sqrt{2}\right)^{2}$.

For calculating $D(\varepsilon)$ we can, as the integrand becomes exponentially small for $y \geq \Re(k) \gg 1$, take the upper bound to infinity, such that it becomes a universal constant. From there we can calculate the conductance in this limit

$$
\bar{G}_{N}(T)=1+B \sqrt{\frac{\varepsilon_{d}}{T}}
$$

where again $B=0.42$ is a universal constant.

These results has a simple physical interpretation. Superconductivity penetrates into the normal part up to $\xi_{N}=\sqrt{\frac{D}{2 \pi T}}$, whereas the rest stays normal, so the total voltage drops over a reduced distance $d-\xi_{N}$. Thus the resistance of the structure is reduced according to the Ohm law. In terms of the conductance, this means

$$
\bar{G}_{N}=1+B^{\prime} \frac{\xi_{N}}{d}
$$

which is equivalent to (23).

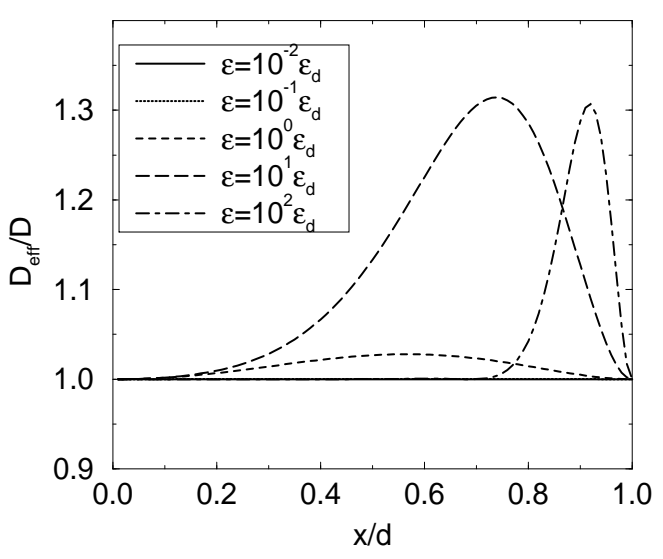

FIG. 3. Local effective diffusion constant

Let us point out, that at both edges of the N-metal the local effective diffusion constant $\mathcal{D}_{\text {eff }}=\cosh ^{2} \theta_{1} \mathcal{D}$ is not enhanced (see Fig. 3) in comparison to its normal state value, because either the Cooper pair amplitude (at the NN' boundary) or the electric field (at the NS boundary) is equal to zero due to the imposed boundary conditions. Inside the $\mathrm{N}$-metal the value $\mathcal{D}_{\text {eff }}$ becomes higher due to nonequilibrium effects in the presence of superconducting correlations $(\eta \neq 0)$. This effect is small at very low energies and becomes more pronounced at $\varepsilon \sim \varepsilon_{d}$.

For temperatures comparable to $\varepsilon_{d}$ the problem was treated numerically. The results show an excellent agreement with our analytical expressions obtained in the corresponding limits. 


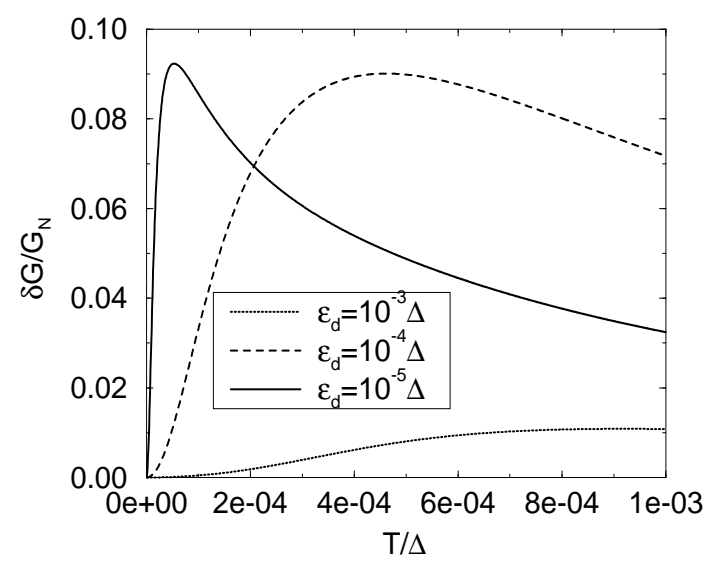

FIG. 4. Conductance in the case of transparent barriers

The numerical results (see fig. 4) confirm, that for $\varepsilon_{d} \ll \Delta$ the universal scaling with $\frac{T}{\varepsilon_{d}}$ is excellently fulfilled, the conductance peak with the height of about $9 \%$ takes place at $T \approx 5 \varepsilon_{d}(\mathrm{cf} 23)$. This peak becomes smaller if we take into account the influence of finite $d_{S}$ keeping $d$ fixed (fig. 5) The qualitative features, however, remain the same.

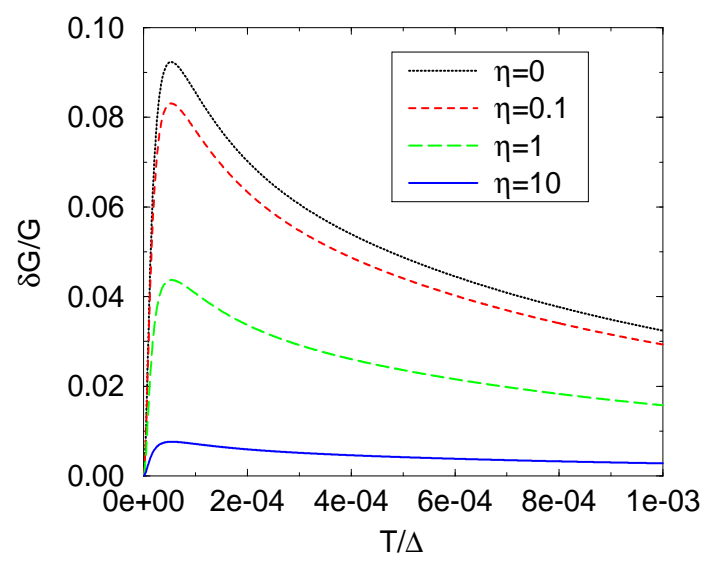

FIG. 5. Conductance normalized to the total length of the normal wire. $\eta:=d_{S} / d, \epsilon_{d}=10^{-5} \Delta$

\section{B. Tunnel barriers}

Let us now assume that a tunnel barrier is present at the $\mathrm{N}^{\prime}-\mathrm{N}$ interface. If one lowers the transparency of this barrier the crossover takes place to the behavior demonstrating monotonously decreasing conductance with T (Fig. 6), which is typical for two serial NIS tunnel junctions. Fig. 6 demonstrates the crossover with increasing $r=\gamma_{B} \xi_{N}^{*} / d$. Inset shows the Arrenius plot for the case of $\gamma_{B} \xi_{N}^{*} / d \gg 1$ which illustrates the activated tunnel-like behavior.

\section{Influence of the barrier at N'N}

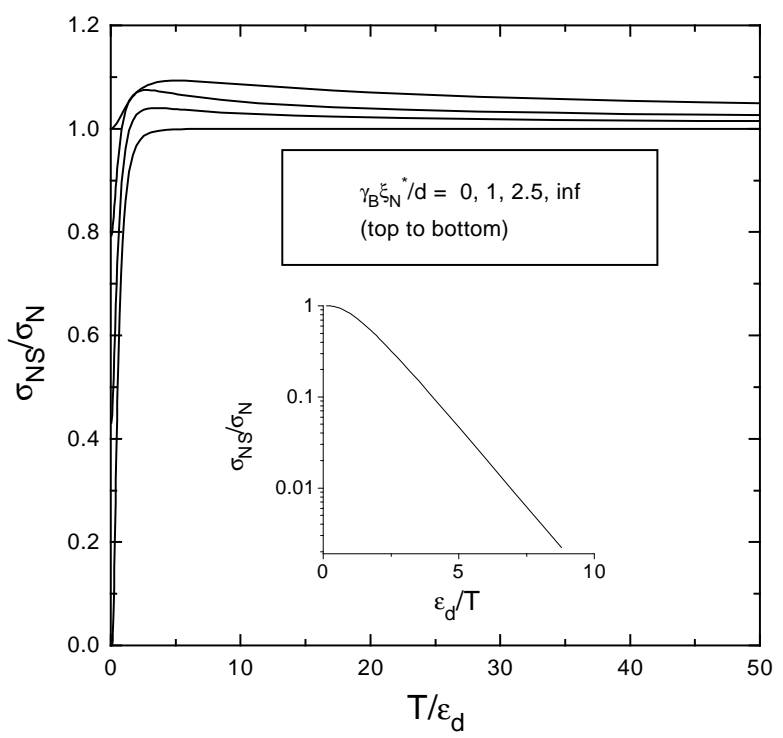

FIG. 6. Conductance in the presence of tunneling barriers

Formally this is due to the term $r / \cosh \theta_{1}(x=$ $0, \varepsilon) \cos \theta_{2}(x=0, \varepsilon)$ in the denominator of Eq. (8), i.e. the contribution of the barrier at the N'-N interface. In the small transparency limit $r \gg 1$ the expression (8) reduces to the standard tunnel formula. The physical reason for this behavior is transparent. For $r \ll 1$ the presence of a tunnel barrier is not important, the electric field penetrates inside the normal metal and we come back to the picture discussed above for perfectly transparent boundaries in which case both normal and correlation DOS play a significant role. If, however, the resistance of a tunnel barrier dominates over the Drude resistance of the normal metal $r \gg 1$, the whole voltage drop is concentrated at the barrier, nonequilibrium effects in the N-metal are absent and therefore only the normal density of states enters into the system conductance.

An additional effect is that a real gap instead of a soft pseudogap develops in the case low transparent tunnel barriers. The crossover between these two regimes is discussed in more detail below.

Note that both types of behavior, namely nonmonotoneous and monotonously decreasing with $\mathrm{T}$ conductance have been observed in the experiments 3 . 


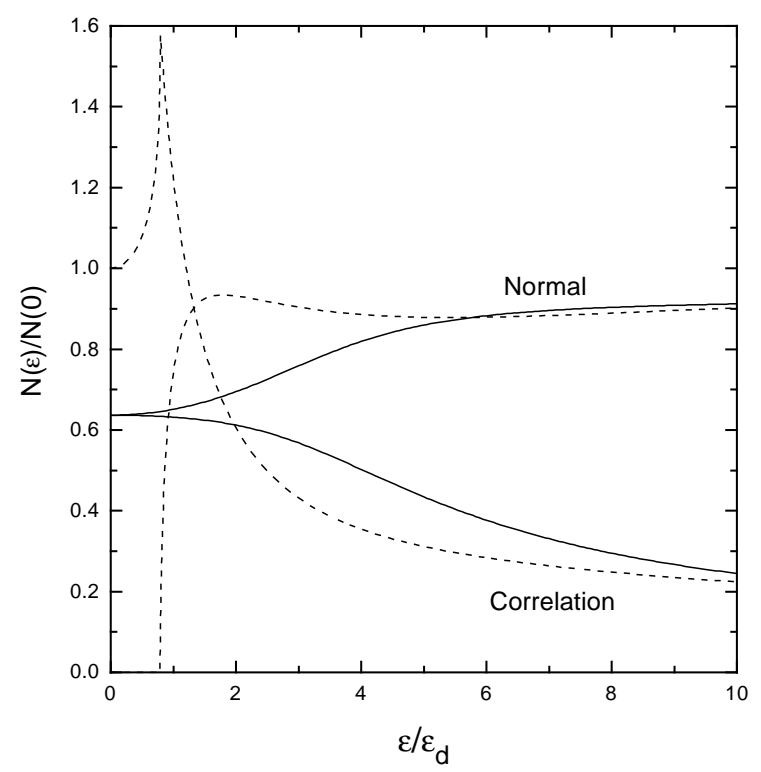

\section{Solid: $\quad$ no barrier at N'-N \\ Dashed: inf high barrier at N'-N}

FIG. 7. Averaged normal and correlation DOS

\section{DENSITY OF STATES}

\section{A. Averaged density}

From our approximative solutions of the preceeding sections, the densities of states can be easily calculated. For $\epsilon=0$ we have $N_{N}=N_{S}=N(0) \frac{2}{\pi}$. At low energies $\epsilon \ll \epsilon_{d}$ there are quadratic corrections: $\quad N_{N / S}=N(0)\left(\frac{2}{\pi} \pm A_{1 / 2}\left(\frac{\epsilon}{\epsilon_{d}}\right)^{2}\right)$ with $A_{1}=$ $\frac{64}{\pi^{5}}\left(1-\frac{8}{\pi^{2}}\right) \approx 0.0396$ and $A_{2}=\frac{16}{\pi^{4}}\left(1+\frac{2}{\pi^{5}}\right) \approx 0.198$. For high energies, the densities approach their normal values, again with square-root corrections $N_{N}=$ $N(0)\left(1-B_{1} \sqrt{\frac{\epsilon_{d}}{\epsilon}}\right)$ and $N_{S}=B_{2} \sqrt{\frac{\epsilon_{d}}{\epsilon}}$ with $B_{1} \approx 0.321$ and $B_{2} \approx 0.75$.

Together with our numerical data (see Fig. 7), this demonstrates the presence of a soft pseudogap in the density of states below the energy $\epsilon_{d}$. Similar results have also been discussed in 22 .
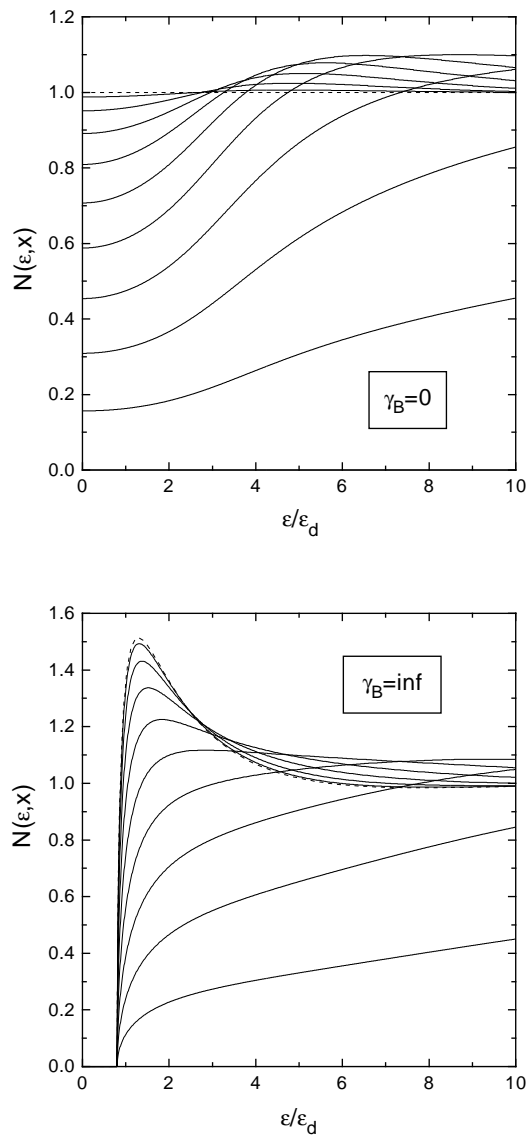

FIG. 8. Local DOS for different $\mathrm{N}-\mathrm{N}^{\prime}$-boundaries. Top: Transparent, Bottom: Non-transparent

\section{B. Spatial dependence and tunneling experiments}

It is also interesting to investigate the spatial dependence of the DOS in the normal layer. Fig. 8 show local normal DOS $N_{N}$ calculated for perfectly transparent $\left(\gamma_{B}=0\right)$ and nontransparent $\left(\gamma_{B}=\infty\right)$ NN' interfaces, respectively, at different distances from the NS boundary: $x / d=0.1,0.2, \ldots, 1$. The difference between these two cases is quite obvious: whereas for $\gamma_{B}=0$ the normal DOS at low energies is always finite, becoming larger at larger values of $x$, for $\gamma_{B}=\infty$ a real gap in the density of states clearly shows up at all energies, Similar results have been recently discussed in Refs 3941 . The overall behavior of the local correlation DOS at each value of $x$ is similar to its average value.

It is important to emphasize that both $\nu(x)$ and $\eta(x)$ are measurable quantities and can be directly probed in experiments. Recently the spatial and energy dependence of the normal DOS has been studied in tunneling 
experiment 42. The data 42 show a qualitative agreement with theoretical predictions. The results obtained here suggest that much better agreement can be achieved if one takes into account smearing of the proximity induced gap in the normal metal due to the diffusion of normal electrons from the external circuit (which plays the role of the N' reservoir) through the NN' boundary. For nontransparent $\mathrm{NN}^{\prime}$ boundaries $\left(\gamma_{B}=\infty\right)$ this process can be neglected and a real gap develops in the N-metal (Fig. 8 (b)). As no such gap was found in 42 we believe that diffusion of normal excitations into the N-layer from the external circuit should play an important role in these experiments. In other words, the experimental situation appears to be closer to that described by the boundary condition $\gamma_{B}=0$ with a soft pseudogap (Fig. 8 (a)) than to the case $\gamma_{B}=\infty$ (see e.g. Ref. 41). The dependence of this effect on the size of the N-layer is depicted in Fig. 9.

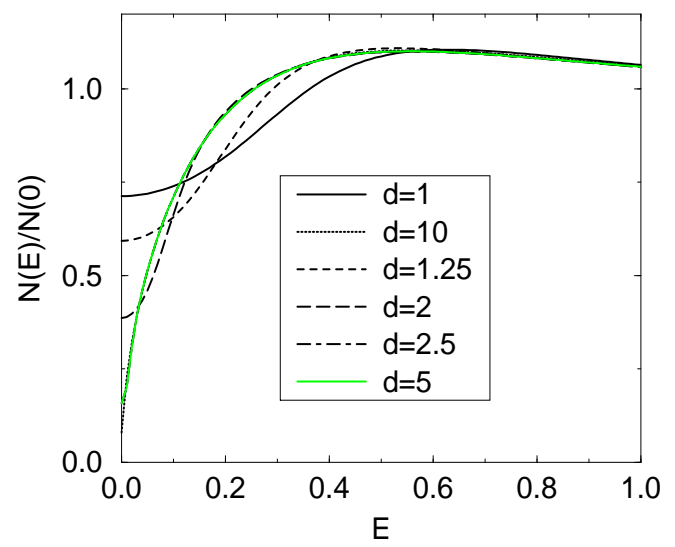

FIG. 9. Size effect on the local normal DOS. Here, the density of states at a fixed distance $x=0.5$ from the $N S$-boundary is plotted for different values of the total length $L$ of the N-part.

Making use of the Usadel equation one can easily recover simple analytic expressions for the density of states at a distance $x_{0}$ away from the NS-boundary. For a Nwire of the total length $d$ at $\epsilon \ll \epsilon_{d}$ we obtain

$$
\frac{N_{N}\left(\epsilon, x_{0}\right)}{N(0)}=\alpha+\frac{\epsilon^{2}}{\epsilon_{d}^{2}} \beta
$$

where $\alpha$ and $\beta$ describe the size effect

$$
\begin{aligned}
& \alpha=\sin \left(\frac{\pi}{2} \frac{x_{0}}{d}\right) \\
& \beta=\frac{32}{\pi^{4}} \alpha\left(1-\frac{x_{0}}{d}-\cos \left(\frac{\pi}{2} \frac{x_{0}}{d}\right)\right)^{2} .
\end{aligned}
$$

Thus for $x_{0} \ll d$ the normal DOS at zero energy and $x=x_{0}$ is proportional to $1 / d$. Neglecting the charging effects (which in principle can also be important 12 ) for the differential conductance of the tunneling probe we find

$$
\left.R_{T} \frac{d I}{d V}\right|_{V=0}=\alpha+\frac{2 \pi^{2}}{3} \frac{T^{2}}{\epsilon_{d}^{2}} \beta
$$

These our results demonstrate that the depairing effect of the N'-reservoir needs to be taking into account on equal footing with pairbreaking due to inelastic scattering 41.42 .

Let us also point out that one can also extract information about the correlation DOS by making two kinds of measurements with the same sample. Indeed, by measuring the conductance of the system (or a part of it) with no tunnel barriers one obtains information about the combination of $N_{N}$ and $N_{S}$ entering the expression for the system condyctance $G$, whereas performing the tunnel experiments 42 one probes only the normal DOS $N_{N}$. Then the correlation DOS can be easily recovered.

\section{ELECTRIC FIELD AND CHARGE}

In this section we shall discuss only the case of perfectly transparent interfaces.

¿From our solutions we can calculate the electric field and the charge by using (11) and the Poisson's equation. The field shows essentially non-monotoneous behavior. At $T=0$ we have $E(\bar{x})=\cos (\bar{x} \pi / 2)-\frac{\pi}{2}(\bar{x}-1) \sin (\bar{x} \pi / 2)$. At high temperatures $T \gg \epsilon_{d}$, the field is constant $E=1$ far from the superconductor where no correlation remains $\left(1-\bar{x} \gg \xi_{\epsilon}\right)$ and it changes linearly near the superconductor: $E(\bar{x})=B_{4}(1-\bar{x}) \sqrt{\frac{T}{\epsilon_{d}}}$ with $B_{4} \approx 2.59$, however, it still overshoots in between these regimes (see Fig. 10).

We see that close to the superconductor the electric field monotoneously decreases with temperature as superconductivity becomes stronger there. Further from the NS boundary the field shows a complicated behavior overshooting the normal state value (the total voltage drop is fixed!) in the region where superconducting correlation starts decaying either due to thermal effects (high $T$ ) or due to the presence of a normal reservoir (low $T$ ). The local resistivity is maximally lowered there and the layer of polarization charges is formed (see Fig. 10). These results emphasize again the importance of nonequilibrium effects for understanding the behavior of the system conductance. 


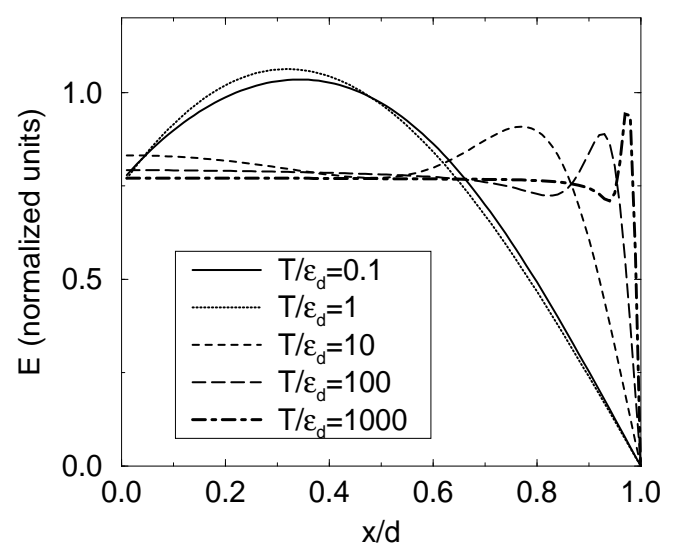

(a) Distribution of the electrical field

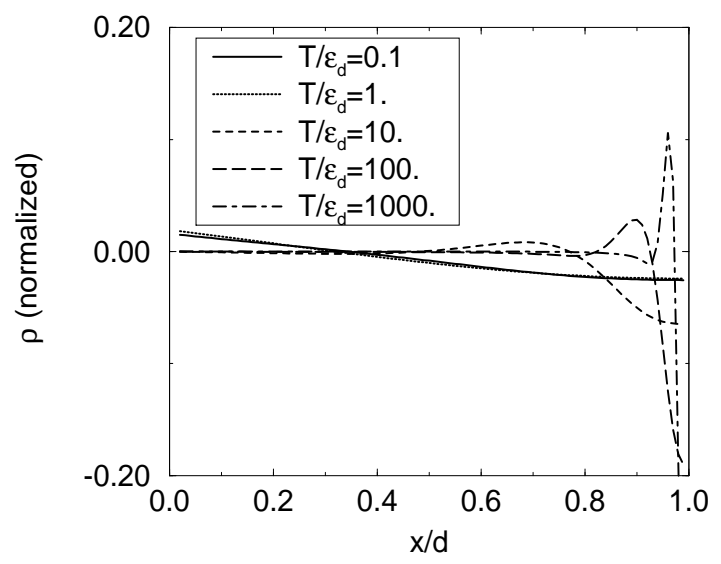

(b) Distribution of the electrical charge

FIG. 10. Electrostatics within the wire

\section{EXTENSION TO SYSTEMS CONTAINING A LOOP}

Recently, the properties of proximity wires foptaining a loop have attracted much experimental 6 and theoretical 44 interest.

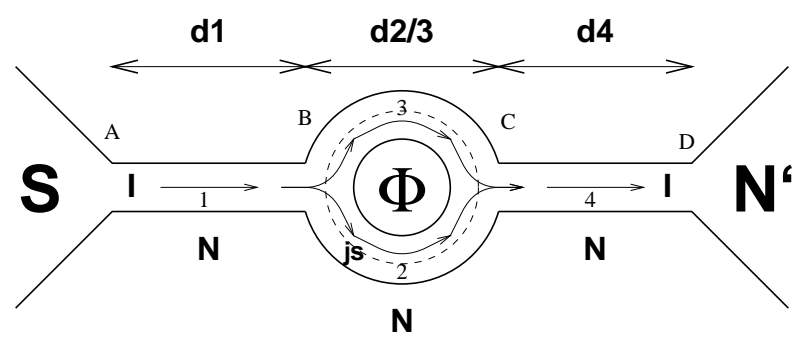

FIG. 11. The system under consideration. $\mathrm{S}$ and $\mathrm{N}$ are respectively superconducting and normal reservoirs. The wire is made of normal conducting material.
If the wire was a real superconductor, the magnetic flux would induce a supercurrent into the ring. As a function of $\Phi$, this current has a period of the superconducting flux quantum $\Phi_{0}=h / 2 e$.

To describe these type of systems, our kinetic scheme has to be extended in several points.

We define the Green's functions in the loop as

$$
G=\cosh u_{\epsilon} \quad F=\sinh u_{\epsilon} e^{i \varphi_{\epsilon}} \quad \varphi_{\epsilon}=\chi_{\epsilon}-2 e \int_{0}^{\vec{x}} d \vec{l} \vec{A}(\vec{l})
$$

where the integration goes along the loop. In the presence of a vector potential, we have to introduce gauge independent derivatives

$$
\nabla \longrightarrow \nabla-2 i e \vec{A}
$$

This means, that instead of solving the Usadel equation with a vector potential, we can perform a gauge transformation and map onto a system without magnetic field having phase $\chi$ instead of $\phi$. As the definition of the Green's functions has to be unique everywhere in the loop, we have $\lim _{x \rightarrow 0+} \varphi(x)=\lim _{x \rightarrow 0-} \varphi(x)(\bmod 2 \pi)$ or

$$
\lim _{x \rightarrow 0+} \chi(x)-\lim _{x \rightarrow 0-} \chi(x)=\frac{2 e \Phi}{\hbar}(\bmod 2 \pi)
$$

after gauge transformation. Here, $\Phi$ is the magnetic flux in the ring.

This mapping shows, that the magnetic field induces a supercurrent $j_{\epsilon}^{S}$ (screening current) into the system. We want to neglect any conversion between this supercurrent and the dissipative current, so both are conserved seperately. This allows the application of the kinetic scheme which has been developed for systems without phase gradient 12 but can be generalized to any system where the dissipative current is conserved.

The Usadel equation then reads 43

$$
\mathcal{D} \frac{d^{2}}{d x^{2}} u_{\epsilon}=-2 i \epsilon \sinh u_{\epsilon}+\frac{\mathcal{D}}{2}\left(\frac{d \chi_{\epsilon}}{d x}\right)^{2} \sinh 2 u_{\epsilon}
$$

and has to be solved together with the equation for the conservation of the supercurrent

$$
\frac{d}{d x} j_{\epsilon}^{S}=0 \quad j_{\epsilon}^{S}=\left|\sinh u_{\epsilon}\right|^{2} \frac{d \chi_{\epsilon}}{d x}
$$

In order to match the Green's functions at branching poits we use the standard continuity condition as no tunnel barriers are assumed to be there. ¿From the Usadel equation in matrix form

$$
D \nabla\left(\check{g_{\epsilon}} \nabla \check{g_{\epsilon}}\right)+i \epsilon\left[\tau_{z}, \check{g_{\epsilon}}\right]=0
$$

follows for any branching point (see also 44 )

$$
\sum_{i=1}^{N} A_{i} \check{g}_{\epsilon} \frac{\partial}{\partial x_{i}} \check{g}_{\epsilon}=0
$$


where the sum runs over matching branches, $\frac{\partial}{\partial x_{i}}$ denotes the derivative in the direction of branch $i$ and $A_{i}$ is the cross-section area of branch $i$. Using our definitions, we get

$$
\sum_{i} A_{i} \frac{\partial u_{\epsilon}}{\partial x_{i}}=0 \quad \sum_{i} A_{i} \frac{\partial \chi_{\epsilon}}{\partial x_{i}}=0
$$

These conditions are equivalent to current conservation, so this is a "Green's functions Kirchhoff law". For $N=1$ Zaitsev's boundary condition 45 for a Normal-Vacuum boundary is reproduced, $N=2$ is equivalent to the trivial statement, that the Green's functions' derivatives are continuous within a branch.

For the calculation of the total transparency $D=1 / m$, we can use the fact, that the $m_{i}$ fulfill Ohm's law just by their definition:

$$
m=\frac{d_{1} m_{1}+d_{4} m_{4}+\left(\frac{1}{d_{2} m_{2}}+\frac{1}{d_{3} m_{3}}\right)^{-1}}{\left(d_{1}+\left(1 / d_{2}+1 / d_{3}\right)^{-1}+d_{4}\right)}
$$

\section{MAGNETORESISTANCE OSCILLATIONS}

The equations (27) and (28) together with boundary conditions (6) and branching conditions (29) have been solved numerically and also analitically in some limiting cases. For numerics, the problem was mapped onto a simpler boundary value problem without any fitting point. As the system of equations is unstable, we used the relaxation method 16 instead of shooting.

For convenience, we have chosen $d_{1}=d_{2}=d_{3}=d_{4}$ and $A_{1}=2 A_{2}=2 A_{3}=A_{4}$, which simplifies the conditions 29. The effect of geometry on the conductance oscillations will be discussed in the appendix. The Thouless energy of just one branch will be labeled as $\epsilon_{d}=\frac{D}{d_{i}^{2}}$.

\section{A. T-dependent Amplitude of $h / 2 e$-Oscillations}

For $T=0$, only quasiparticles with the energy $\epsilon=0$ contribute to the conductance. From 27 and 6 we can conclude, that $u_{\epsilon=0}$ is a purely imaginary function, so the total conductance of the system is equal to its normal state value, being independent of $\Phi$. In other words, there exist no conductance oscillations at $T=0$ (cf. 88.47 ).

At nonzero temperatures the system conductance depends on the magnetic flux inside the loop with the period equal to the flux quantum $\Phi_{0}$ (see fig. 12). With the aid of simple analytic arguments (see Appendix) one can conclude that at low temperatures the amplitude of the conductance oscillations increases as $T^{2}$ (see fig. 13).

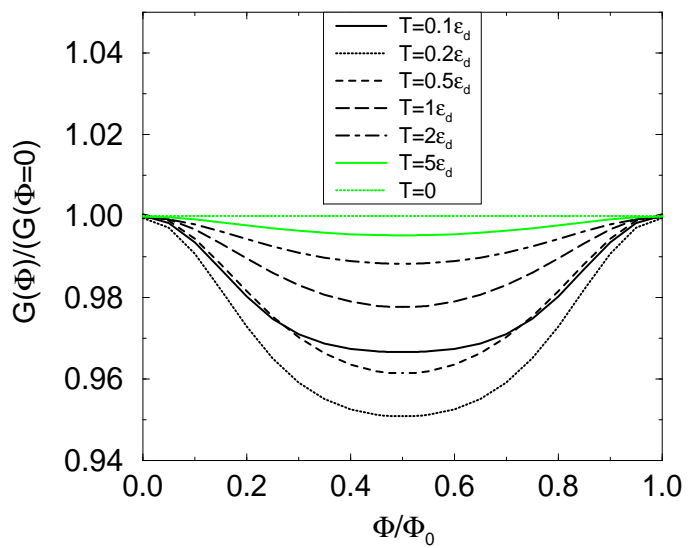

FIG. 12. $h / 2 e$-periodic structure of the conductance, normalized to the zero-field conductance

In order to establish the temperature dependence of this amplitude at higher $T \gg \epsilon_{d}$ it is convenient to make use of the fact that for electrons with sufficiently large energies $\epsilon \gtrsim \epsilon_{d}$ superconducting correlation is destroyed already before they reach the loop. Thus at such energies the transparency of the whole structure $D(\epsilon)$ should be insensitive to the particular value of the flux inside the loop. In other words, calculating the flux-dependent part of the system conductance we can take into account only the contribution of low energy quasiparticles which remain correlated in the loop area. E.g. for the amplitude of the conductance oscillations we get

$$
\begin{aligned}
\Delta G(T) & =G_{h / 4 e}(T)-G_{0}(T) \\
& =\frac{1}{2 T} \int_{0}^{\infty} d \epsilon\left(D_{h / 4 e}(\epsilon)-D_{0}(\epsilon)\right) \operatorname{sech}^{2}(\epsilon / 2 T) \\
& \approx \frac{1}{2 T} \int_{0}^{\epsilon_{c}} d \epsilon\left(D_{h / 4 e}(\epsilon)-D_{0}(\epsilon)\right) \operatorname{sech}^{2}(\epsilon / 2 T) \\
& \approx \frac{\epsilon_{c}}{2 T} \Delta D_{a v}
\end{aligned}
$$

where $\epsilon_{c}$ is the cutoff parameter of order $\epsilon_{d}$, and $\Delta D_{a v}$ is constant.

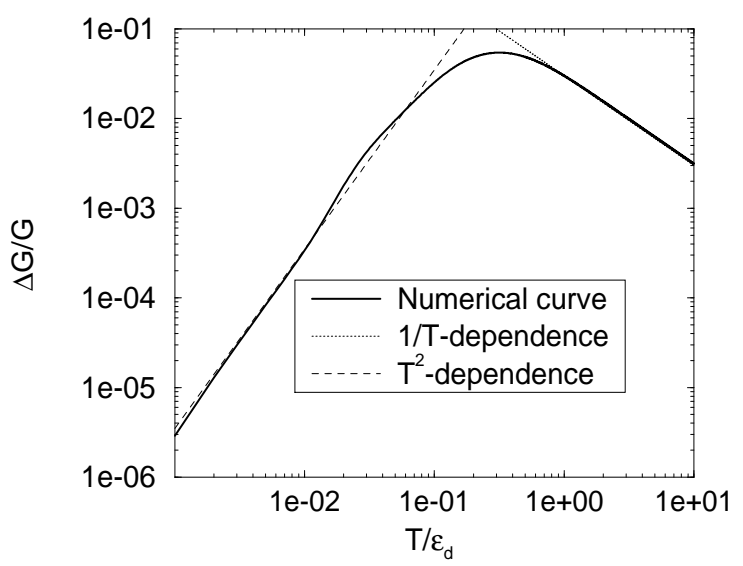

FIG. 13. Oscillation amplitude 


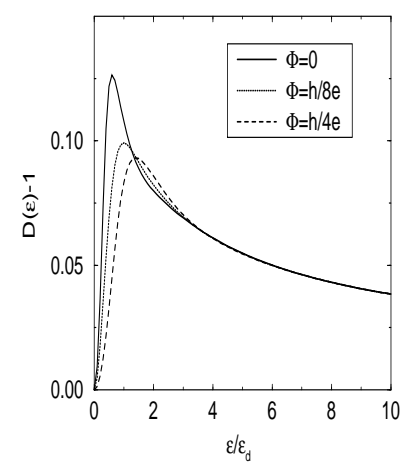

(a) Transparency

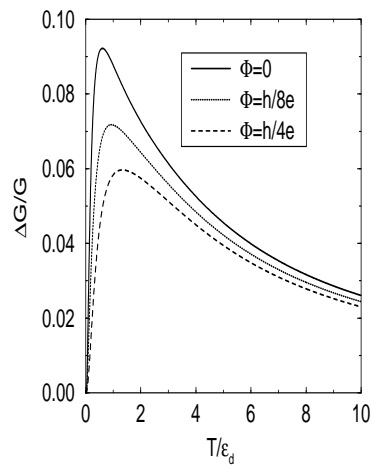

(b) Conductance
FIG. 14. Temperature dependence of tranport properties at different fluxes

The results of our numerical analysis fully support the simple analytic arguments presented above. The system transparency $D(\epsilon)$ is depicted in Fig. 14 (a) for different values of the flux $\Phi$. The value $D(\epsilon)$ depends on $\Phi$ only at low energies, whereas for $\epsilon \gtrsim \epsilon_{d}$ all curves merge. In accordance to our simple estimate (30) this leads to a $1 / T$ decay of the oscillation amplitude $\Delta G$ at large $T$ (see Figs. 14 (b) and 12). Also the $T^{2}$ behavior of $\Delta G$ in the low temperature limit is recovered (Fig. 12).

The $1 / T$ behavior of $\Delta G$ has been also found in recent experiment \$6. We would like to point out that a slow power-law decay of the conductance due to a dominating contribution of low energy quasiparticles just emphasizes the physical difference between kinetic and thermodynamic quantities, like supercurrent which decays exponentially with increasing $T$.

\section{B. Flux-Dependent DOS}

As it was already discussed the simultaneous presence of correlated electrons and the electric field in the normal metal causes nontrivial nonequilibrium effects, the description of which involves two densities of states $\nu(x)$ and $\eta(x)$. In the presence of the normal metal loop with the magnetic flux $\Phi$ in our system there appeas a possibility to tune both normal and correlation DOS by changing the value of $\Phi$. For the system depicted in Fig. 11 these densities of states can be easily calculated. As one might expect for the region between the superconductor and the loop (between the points A and B) this dependence is quite weak and both DOS practically coincide with those calculated above for a wire without the loop. On the other hand, in the region between the loop and the normal reservoir $\mathrm{N}^{\prime}$ (between the points $\mathrm{C}$ and $\mathrm{D}$ ) the quantities $\nu(x)$ and $\eta(x)$ are very sensitive to the flux $\Phi$.

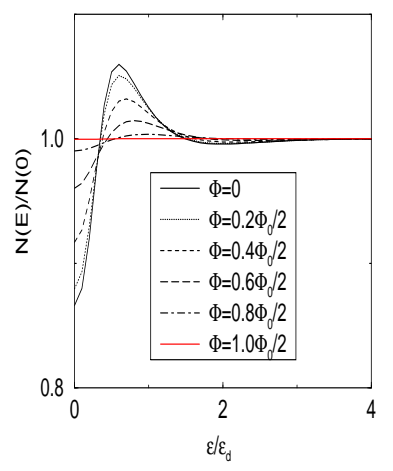

(a) Normal

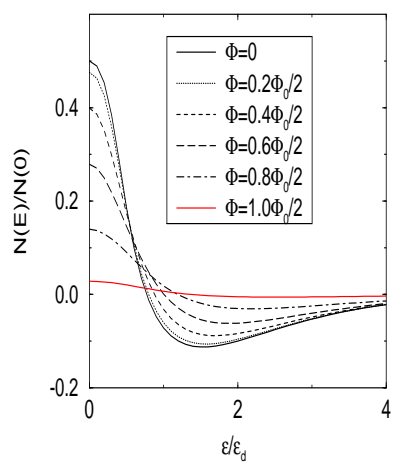

(b) Correlation
FIG. 15. Flux-dependence of the two densities of states in point $\mathrm{C}$ of the system

The normal and correlation DOS for the point $\mathrm{C}$ (Fig. 11) are presented in Fig. 15. We see that with increasing the value of the magnetic flux the proximity induced pseudogap decreases and vanishes completely as the flux approaches the value $\Phi_{0} / 2$. For such value of $\Phi$ the proximity effect in the region "after" the loop is completely destroyed, the pseudogap is fully suppressed and the normal DOS coincides with $N(0)$ at all energies. Accordingly the correlation DOS vanishes at $\Phi=\Phi_{0} / 2$. Thus in this case the resistance of the region between the points $\mathrm{C}$ and $\mathrm{D}$ is equal to its normal state value at all $T$.

These results demonstrate that "the strength" of the proximity effect in our system can be regulated by the external magnetic flux. This might serve as an additional experimental tool for investigation of proximity induced superconductivity in normal metalic structures. In particular we believe that it wuld be interesting to repeat the tunneling experiments 42 in the presence of the loop with the magnetic flux. Such experiments would provide a direct probe of the dependence of the densities of states on $\Phi$ (Fig. 15 (a)).

\section{SUMMARY AND OUTLOOK}

We have used a microscopic kinetic analysis to describe the transport properties of superconductor-normal metal proximity structures. In the case of transparent intermetallic boundaries we demonstrated a reentrant behavior of the system conductance with temperature. This behavior was attributed to nonequilibrium effects occuring in the normal metal in the presence of proximity induced superconductivity and the electric field. We argued that under these conditions both uncorrelated ("normal") and correlated ("superconducting") electrons contribute to the system conductance which is henceforth defined by a combination of two densities of states - the normal DOS $N_{N}$ and the correlation DOS $N_{S}$. The latter is known 
to play an important role whenever the quasiparticle distribution fumction in a superconductor is driven out of equilibriumes.

We studied the energy dependence of both these quantities and demonstrated that if the normal metal is brought in a direct contact to a superconductor on one side and a big normal reservoir N' on the other side the normal DOS $N_{N}$ shows a soft pseudogap at energies below the Thouless energy $\epsilon_{d}$. This effect is due to an interplay between correlated and uncorrelated electrons penetrating into the N-layer respectively from a superconductor and a normal reservoir. If a low transparency tunnel barrier is present at the NN' interface the diffusion of normal excitations into the $\mathrm{N}$-metal is suppressed, the influence of a superconductor prevails and a real gap in the density of states develops.

Low transparent tunnel barriers also prevent the electric field from penetration into the N-layer thus suppressing nonequilibrium effects there. We demonstrated that with the aid of a proper combination of the systems with and without tunnel barriers one can directly probe both energy and spacial dependencies of both densities of states $N_{N}$ and $N_{S}$ in one experiment.

We extended our analysis to proximity systems containing the normal metal loop with the magnetic flux $\Phi$. We demonstrated that the conductance of such systems as a function of $\Phi$ oscillates with the period equal to the flux quantum $\Phi_{0}=h / 2 e$. The amplitude of these oscillations $\Delta G$ also shows the reentrant behavior being equal to zero at $T=0$, increasing as $T^{2}$ at $T \lesssim \epsilon_{d}$ and decaying as $1 / T$ at $T \gtrsim \epsilon_{d}$. We argued that even at high temperatures $T \gg \epsilon_{d}$ low energy electrons with $\epsilon \lesssim \epsilon_{d}$ are only responsible for the conductance oscillations leading to the power law decay of $\Delta G$ at large $T$. We pointed out that the densities of states $N_{N}$ and $N_{S}$ can be tuned (decreased) by applying the magnetic flux $\Phi$. In particular, if the flux in the loop is equal to the half of the flux quantum $\Phi=\Phi_{0}$ the proximity effect in the region "after" the loop is completely suppressed, the normal DOS $N_{N}=N(0)$ at all energies and $N_{S}=0$. This effect can be also directly probed in tunneling experiments and used for further studies of proximity induced superconductivity in normal metallic systems.

We acknowledge useful discussions with C.Bruder, W.Belzig, H.Courtois, D.Esteve, B.Pannetier, V.T.Petrashov, G.Schön, B.Spivak, A.F.Volkov and B.J. van Wees. This work was supported by the Deutsche Forschungsgemeinschaft within the Sonderforschungsbereich 195. A.A.G acknowledges partial support by RFFI No.96-02-1956.

\section{APPENDIX A: A CLOSER LOOK AT THE H/2E-OSCILLATIONS}

Here we present further details related to the effect of geometry on the behavior of the proximity NS systems containing a mesoscopic normal metal loop with the magnetic flux (see Fig. 11). In the first three sections we will keep $A_{1}=2 A_{2}=2 A_{3}=A_{4}$ for simplicity allowing different values for the $d_{i}$ but restricting ourself to symmetric loops $d_{2}=d_{3}$.

\section{Low temperature behavior}

For examining the low energy range, which is dominant for the conductance oscillations at any temperature as stated above, we proceed perturbatively from the case $\epsilon=0, \phi=0$ first to finite flux, then to finite $\epsilon$. As the Usadel equation is quadratic in $\frac{d \chi}{d x}$, the value $u$ does not distinguish between the upper and the lower branches of the ring (2 or 3 in Fig. 11), so we will not make a difference in the notation.

We start from $\epsilon=0, \Phi=0$, where $u$ is purely imaginary and therefore yields $D=1$. For a finite value of the flux but $\epsilon=0$, we get a purely imaginary correction and therefore $D_{\phi, 0}=1$. This correction is quadratic in the flux as the r.h.s. of the equation is quadratic in the phase. The finite energy correction at zero flux is a real function and is quadratic in $\epsilon$.

Thus proceeding perturbatively we find $\Re u \propto \epsilon \Phi^{2}$, and from the expansion

$$
\begin{aligned}
u= & -i \frac{\pi x}{2 d_{\Sigma}}+i \phi^{2} g_{d_{1}, d_{2}, d_{3}, d_{4}}(x)+\epsilon h_{d_{1}, d_{2}, d_{3}, d_{4}}(x) \\
& +\epsilon \phi^{2} k_{d_{1}, d_{2}, d_{3}, d_{4}}(x)+i \epsilon \phi^{2} l_{d_{1}, d_{2}, d_{3}, d_{4}}(x)
\end{aligned}
$$

with $d_{\Sigma}=d_{1}+d_{2}+d_{4}$, in the leading order we get

$$
\begin{aligned}
D(\epsilon) & =\left(\frac{1}{d_{\Sigma}} \int_{0}^{d_{\Sigma}} \frac{d x}{\cosh ^{2}\left(u_{1}(x)\right)}\right)^{-1} \\
& =\left(\frac{1}{d_{\Sigma}} \int_{0}^{d_{\Sigma}} d x\left(1-\frac{\epsilon^{2}}{2}\left(h+\Phi^{2} k\right)^{2}\right)+O\left(\epsilon^{4}, \phi^{4}\right)\right)^{-1} \\
& =\frac{1}{d_{\Sigma}}\left(1+\frac{\epsilon^{2}}{2}\left(\lambda_{d_{1}, \ldots, d_{4}}-\phi^{2} \mu_{d_{1}, \ldots, d_{4}}\right)+O\left(\epsilon^{4}, \phi^{4}\right)\right.
\end{aligned}
$$

where the coefficients are defined as

$$
\begin{aligned}
& \lambda=\int_{0}^{d_{\Sigma}} d x h^{2}(x) \\
& \mu=4 \int_{0}^{d_{\Sigma}} d x h(x) k(x) .
\end{aligned}
$$

Thus at low $T$ both the transparency and the conductance depend quadratically on energy and flux. Further analytic expressions are presented in 4 .

It is remarkable, that for $d_{3}=0$ due to (6) we have $\sinh u=0$ at the point $C$. Therefore, the current conservation condition (28) can be fulfilled for $j=0, \chi= \pm \phi / 2$ 
(different signs refer to different branches), so the phase gradient is zero almost everywhere and the Usadel equation does not contain the phase any more. Thus no magnetoresistance oscillations occur in this case.

\section{Cutoff energy}

Let us estimate the cutoff energy $\epsilon_{c}$.

Consider the case $\epsilon \gg \epsilon_{d_{1}+d_{2}}=\frac{{ }^{D}}{\left(d_{1}+d_{2}\right)^{2}}$. For $\Phi=0$ we again have (21) $u=4 \arctan \left(\frac{i \pi}{8} e^{-k^{R} x}\right)$, so for small $\phi$ we can proceed perturbutavely. As the supercurrent is exponentially small, we can approximate the phase profile as

$$
\frac{d \chi}{d x}=\frac{1}{2 \sinh k_{1} d_{2}} e^{k_{1}\left(x-d_{1}-d_{2} / 2\right)},
$$

so the influence of the magnetic flux is concentrated within a distance $\max \left\{\xi_{N, \epsilon}, d_{2}\right\}$ from the point $C$ (see Fig. 11). However, as $u$ is exponentially small there, the oscillations of the transparency are exponentially surpressed, so we can estimate $\epsilon_{c} \leq \epsilon_{d_{1}+d_{2}}$, which depends only on the sum of these length, but not on $d_{1}$ alone (see also fig. 16.).

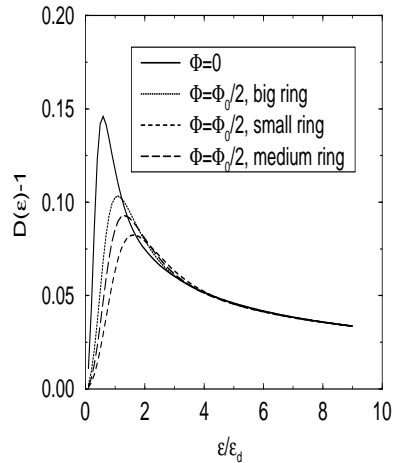

(a) Transparency

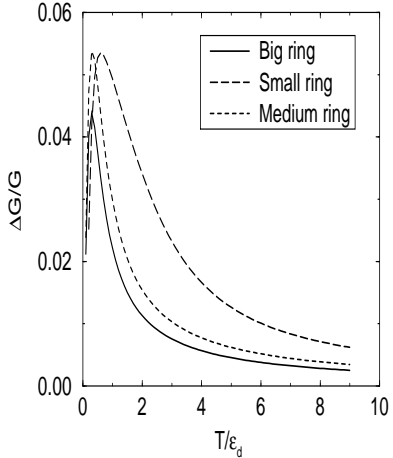

(b) Amplitude of conductance oscillations
FIG. 16. Size effects onto the conductance oscillations. Small: $d_{1}=d_{4}=1.2, d_{2}=d_{3}=0.6$, Medium: $d_{1}=d_{2}=d_{3}=d_{4}=1$, Big: $d_{1}=d_{4}=0.8$, $d_{2}=d_{3} 1.4$.

The key feature of Fig. 16 (b) is the strong fluxdependence for systems with small rings. This fact can be also recovered from the Usadel equation: for $d_{2} \ll d$ one estimates $\frac{d \chi}{d x} \approx \frac{\Phi}{2 \Phi_{0} d_{2}}$. As this enters quadratically and in the end we have to integrate over the ring only once, the contribution of the ring is roughly $\propto 1 / d_{2}$.

\section{Cross section effect}

For the sake of simplicity above we have sticked to the case $2 A_{2}=A_{1}=A_{3}$. As this condition might not be fulfilled in real experiments it is worthwhile to check whether the main features of our analysis survive for other values of $A_{1,2,3}$. In order to do that we performed calculations also for the case $A_{1}=A_{2}=A_{3}$. The results are similar to those obtained before, showing an additional dip structure in the transparency at intermediate energies (Fig. 17(a)) and a slightly deformed $G$ in the same energy interval ( Fig. 17(b)).

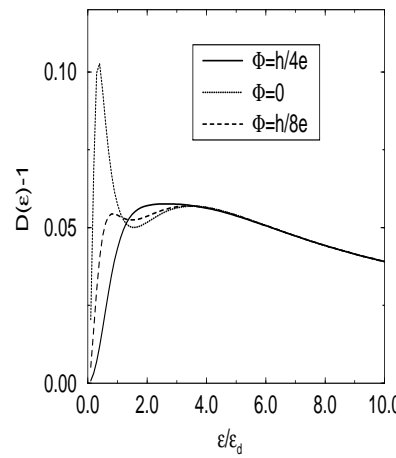

(a) Transparency

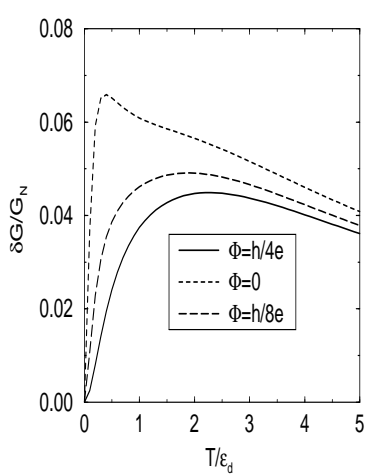

(b) Conductance
FIG. 17. Transport quantities for the system with $A_{1}=A_{2}=A_{3}=A_{4}$ and $d_{1}=d_{2}=d_{3}=d_{4}$ displaying the cross-section effect.

${ }^{1}$ P.G. de Gennes. Superconductivity of metals and alloys.

${ }^{2}$ A.Kastalsky et al., Phys. Rev. Lett. 67, 3026 (1991).

${ }^{3}$ V.T.Petrashov et al., Phys.Rev. Lett. 74, 5268 (1995).

${ }^{4}$ H.Pothier et al., Phys. Rev. Lett. 73, 2488 (1994).

${ }^{5}$ H. Courtois et al., Phys. Rev. B 52, 1162 1995).

${ }^{6}$ H. Courtois et al., Phys. Rev. Lett. 76, 130 (1996).

${ }^{7} \mathrm{H}$. Courtois et al., to appear in the proceedings of "Rencontres de Moriond 1996"

${ }^{8}$ P.Charlat et al., submitted to Phys. Rev. Lett.

${ }^{9}$ A.V.Zaitsev, JETP Lett. 51, 41 (1990).

${ }^{10}$ A.F.Volkov, JETP.Lett. 55, 747 (1992).

11 A.F.Volkov, Phys. Lett. 187, 404 (1994).

12 A.F.Volkov, A.V.Zaitsev, and T.M.Klapwijk, Physica C 210, 21 (1993).

13 C.W.J.Beenakker, Phys.Rev.B 46, 12841 (1992).

${ }^{14}$ C.W.J.Beenakker, B.Rejaei, and J.A.Melsen, Phys.Rev.Lett. B 72, 2470 (1994). 
${ }^{15}$ F.W.J.Hekking and Yu.V.Nazarov, Phys.Rev.Lett. 71, 1625 (1993).

${ }^{16}$ Yu.V.Nazarov, Phys.Rev.Lett. 73, 134 (1994).

17 A.D.Zaikin, Physica B 203, 255 (1994)

${ }^{18}$ F.Zhou, B.Spivak and A.Zyuzin, Phys. Rev. B 52, 4467 (1995).

19 S. Yip, Phys. Rev. B 52, 15504 (1995).

${ }^{20}$ A.F.Volkov, N.Allsopp, and C.J.Lambert, J. Phys. Cond. Mat. 8, L 45 (1996).

${ }^{21}$ F.Wilhelm, G.Schön, and A.D.Zaikin, in preparation.

${ }^{22}$ A.A.Golubov and M.Yu.Kupriyanov, Sov. Phys. JETP 69, 805 (1989); JETP Lett. 61, 855 (1995).

${ }^{23}$ Yu. V. Nazarov and T.H. Stoof, Phys. Rev. Lett. 76, 823 (1996); Phys. Rev. B 53, 1050 (1996).

24 S.N.Artemenko, A.F.Volkov, and A.V.Zaitsev, Sol. St. Comm. 30, 771 (1979).

25 see e.g. A. Schmid, in Nonequilibrium Superconductivity, Phonons, and Kapitze Boundaries, ed. K.E. Gray, (Plenum, N.Y.,1981), p. 423.

26 This argument still does not explain why at $T=0$ the system conductance is exactly equal to its normal state value. Unlike in the case of ballistic NS constrictions (where a transparent physical picture for the doubling of the linear conductance due to Andreev reflections was presented27) for diffusive NS structures a simple physical interpretation of the above result - if it exists at all - is still lacking.

27 G.E.Blonder, M.Tinkham, and T.M.Klapwijk, Phys.Rev.B 25, 4515 (1982).

${ }^{28}$ A.D.Zaikin, Zh. Eksp. Teor. Phys. 84, 1560 (1983) [Sov. Phys. JETP (1983)]; U.Gunsenheimer, and A.D.Zaikin, Phys. Rev. B 50, 6317 (1994).

29 see e.g. B.J. van Wees, P. de Vries, P. Magnée and T.M. Klapwijk, Phys. Rev. Lett. 69, 510 (1992).

${ }^{30}$ G.M.Eliashberg, Sov.Phys.JETP 34, 668 (1971).

31 A.I.Larkin, and Yu.N.Ovchinnikov, Sov.Phys.JETP 41, 960 (1975).

${ }^{32}$ M.Yu.Kupriyanov, and V.F.Lukichev, Sov. Phys. JETP 67, 1163 (1988).

${ }^{33}$ A.F.Volkov, P.H.C.Magnee, B.J. van Wees, and T.M.Klapwijk, Physica C 242, 261 (1995).

${ }^{34}$ see e.g. J.Beyer,C.J.Pethik, and J.Rammer, in: Nonequilibrium Superconductivity, eds. D.N. Langenberg and A.I.Larkin (North Holland, Amsterdam, 1986), p. 129.

35 A.Schmid and G.Schön, J.Low.Temp.Phys. 20, 207 (1975).

${ }^{36}$ M.Yu.Kupriyanov, Sov. J. Superconductivity 2, 5 (1989).

37 A.A.Golubov, F.K.Wilhelm, and A.D.Zaikin, preprint cond-mat 9601085 .

38 A.D.Zaikin, F.K.Wilhelm, and A.A.Golubov, to appear in the Proceedings of the "Rencontres de Moriond" Conference (1996).

39 B.A.Aminov, A.A.Golubov, and M.Yu.Kupriyanov, Phys. Rev. B 53, 365 (1996).

40 A.A.Golubov, et al., Phys. Rev. B 51, 1073 (1995).

${ }^{41}$ W.Belzig, C.Bruder, and G. Schön, Submitted to Phys. Rev. B..

${ }^{42}$ S. Guéron et al., submitted to Phys. Rev. Lett.

${ }^{43}$ A.D.Zaikin, and G.F.Zharkov, Fiz. Nizk. Temp. 7, 375 (1981)[Sov. J. Low Temp. Phys.]; A.D.Zaikin, in: Nonequilibrium Superconductivity, ed. V.L.Ginzburg (Nova Science,
N.Y., 1986), p. 57

${ }^{44}$ A.V. Zaitsev, Physica B 203, 274 (1994).

${ }^{45}$ A.V. Zaitsev, Sov.Phys. JETP 59, 1015 (1984).

${ }^{46}$ W. H. Press et al., Numerical Recipes in FORTRAN (Cambridge University Press, 1992), p. 753-764.

${ }^{47} \mathrm{Yu}$. V. Nazarov and T.H. Stoof, preprint.

${ }^{48}$ F.K.Wilhelm, thesis "Diplomarbeit", available on request. 